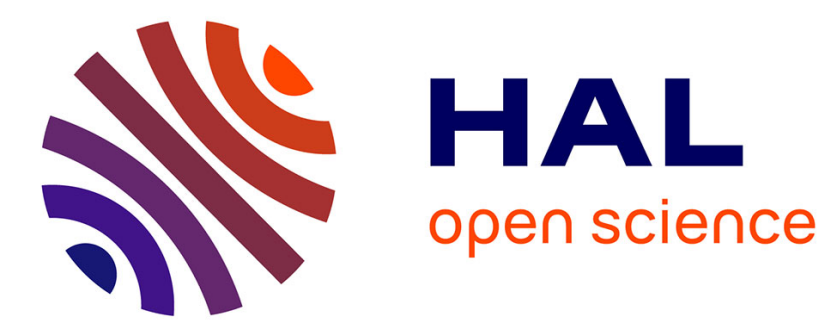

\title{
Les espaces zonards : vers une observation collaborative
} Tristana Pimor

\section{To cite this version:}

Tristana Pimor. Les espaces zonards: vers une observation collaborative. Espaces et sociétés (Paris, France), 2016, L'observation et ses angles, 1-2 (164-165). hal-01287196

\section{HAL Id: hal-01287196 https://hal.science/hal-01287196}

Submitted on 12 Mar 2016

HAL is a multi-disciplinary open access archive for the deposit and dissemination of scientific research documents, whether they are published or not. The documents may come from teaching and research institutions in France or abroad, or from public or private research centers.
L'archive ouverte pluridisciplinaire HAL, est destinée au dépôt et à la diffusion de documents scientifiques de niveau recherche, publiés ou non, émanant des établissements d'enseignement et de recherche français ou étrangers, des laboratoires publics ou privés. 
Tristana Pimor,

Maîtresse de conférences en sciences de l'éducation, Université Paris Est Créteil, département carrières sociales, laboratoire LIRTES, OUIEP.

Coordonnées personnelles: 19 rue de Ferland, 77133 Féricy (veuillez m'adresser les courrier à celle-ci, s’il vous plait)

Coordonnées professionnelles : Dpt Carrières sociales, IUT Sénart, Fontainebleau, UPEC, 3 6-37 rue Georges Charpak, 77127 Lieusaint.

Titre de l'article : Les espaces zonards : vers une observation collaborative

\section{0 signes}




\section{Les espaces zonards : vers une observation collaborative}

Mots clefs : Ethnographie collaborative, squat, jeunes en errance, rue, observation participante

Résumé :

À partir d'une recherche ethnographique réalisée sur les pratiques délinquantes de jeunes de la rue qui se définissent eux-mêmes comme "zonards », une série d'interrogations sur les postures d'observation (distanciée, participante, collaborative) va progressivementémerger des espaces où se déroulent l'enquête et reconfigurer la posture de l'observateur. Cet article souligne les effets du recours à l'induction, et les redéfinitionsméthodologiques que nos contextes d'enquête nous imposent. Ils peuvent constituer tout autant des freinsque des sources d'innovationsméthodologiques et heuristiques. L'attention sensible portée au terrain zonard d'où l'observation collaborative avec les zonards enquêtés découle permet ainsi d'obtenir des données intimes, plus proches du vécu des acteurs sur leursparcours biographiques.

Key words: collaborative ethnography, squat, young homeless, street, participant observation

\section{Abstract :}

Starting with an ethnographical research on young homeless delinquent practices, spaces fieldwork made emerge observation posture questioning(distanced, participant, collaborative). This article showsthe influence of induction approachand the methodological redefinings adaptation imposed by our space fieldwork They can constitute just as much brakes than sourcesof methodological and heuristics innovations. The sensitive attention carriedin zonard fielwork where the collaborative observation is ensue from allows to obtain intimate data, closer to the real-life experience of the actors.

Palabras llaves : Etnografía colaborativa, vivienda ocupada ilegalmente, jóvenes en vagabundeo, calle, observación participante

\section{Resumen :}

A partir de una investigación etnográfica realizada sobre las prácticas delincuentes de jóvenes de la calle que mismos se define como "zonards ", una serie de interrogaciones sobre las posturas de observación (distanciada, participantes, colaborativas) va a emerger de espacios donde se celebran la encuesta y reconfigurar la postura del observador. Este artículo subraya los efectos del recurso a la inducción, y las redefiniciones metodológicas que nuestros contextos de investigación nos imponen. Pueden constituir tanto frenos como fuentes de innovaciones metodológicas y heurísticas. La atención sensible llevada al terreno zonard de donde la observación colaborativa con zonards investigado emana permite así obtener datos íntimos, más próximos del vivido de los actors. 
Si les thèses ethnographiques explicitent la conduite d'observations, dans les ouvrages publiés, les ethnographes relatent peu la manière dont ils l'ont menée- et ce, malgré l'ouverture d'une brèche par J-Favret-Saada dans « La mort, les mots, les sorts » (1985). Préférant sacrifier la description de l'observation, supputéetrop narcissique et soporifiquepour nos lecteurs en faveur de nos analyses, nous alimentons sans nous en rendre compte la suspicion quant à la "rigueur du qualitatif ${ }^{1}$ et aux qualités heuristiques de cet outil. C'est pourtant dans cet exercice du terrain, soumis à l'incertitude que des réorientations méthodologiques et théoriques s'opèrent pour s'accorder davantage à ce et ceux que l'on rencontre.L'expliquer n'est ainsi pas accessoire et autorise le lecteur à suivre la genèse de nos interprétations, ce quilui permet d'évaluer la pertinence de nos propositions et d'y participer (Marcus, Clifford, 1986). Ici, la description analytique d'observations menées dans le cadre de mémoires universitaires et d'une thèse ${ }^{2}$ portant sur le mode de vie et les parcours biographiques ${ }^{3}$ d'un groupe de jeunes en errance permetde saisir en quoi les espaces d'observation sont sources de contraintes et d'innovations en matière d'observation et d'interprétation. Le concept d'espace renvoie ici à des lieux, à l'espaceplus métaphorique de l'errance juvénile ${ }^{4}$ qui se distingue des recherches sur les SDF.En partant de cette recherche sur une jeunesse de la rue qui se qualifie elle-même de « zonarde » ou de «zone », terme indigène qui désigne la communauté zonarde de la ville d'enquête et leur territoire de socialité situé dans le centre-ville, il sera question de comprendre en quoi certainesrecherches sur cette jeunesse,mon expérience d'éducatrice, leur mode de vieont délimité ma façon d'observer et d'entendre ce qu'est devenir et être «zonard». J'expliquerai commentla fréquentation d'une famille de rue $e^{5}$ dans des espaces spécifiques d'observation m'a poussé à adopter une posture d'observatrice plus impliquée, à mettre en place une recherche collaborative, à diversifier les populations de l'enquête et les modes d'observer afin d'établir des conclusions originales sur les parcours biographiques zonards.

\section{JEUNES EN ERRANCE : UN REGARD, UN ESPACE D'ENFERMEMENT METHODOLOGIQUE}

M-Blondet (in Bensa, Fassin, 2008) évoque l'importance d'effectuer non pas seulement une rupture épistémologique avec les catégories du sens commun, mais aussi d'interroger les représentations enfermantes que nos disciplines et champs de recherche créent. L'héritage académique agit comme un filtre ethnocentriquequi programme notre regard,notre façon de concevoir nos outils et notre posture de chercheur (Kuhn, 1962). La contextualisation des travaux antérieurs aux nôtres sur nos thèmes de recherche se révèle indispensable pour identifier les représentationsqui colorent les façons d'effectuer des observations et d'interpréter nos données.

\footnotetext{
${ }^{1}$ Titre de l'ouvrage de J-P Olivier de Sardan (2008)

${ }^{2}$ T. Pimor, 2013, En famille dans la rue, Université de Bordeaux.

${ }^{3}$ Le parcours est un ensemble de trajectoires en interrelation. Les trajectoires sont familiale, scolaire, professionnelle, amicale... (Grossetti et al, 2010)

${ }^{4}$ Entendu comme le regard que délivre les écrits produits par le travail social et la sciences sociales qui adoptent cette terminologie.

${ }^{5}$ Groupe de zonards ayant des liens affectifs forts qui se nomment eux-mêmes « La Family ».
} 
Les zonards avec qui j'ai réalisé le travail de terrain sont couramment désignés comme «jeunes en errance ». Ces termes sont apparus en 1991 sous la plume de F. afin de définir une catégorie de jeunes alors non identifié(Chobeaux, 2001). Pris dans des logiques de rentabilité, de recherche de subvention, le travail social trouva dans cette terminologie un moyen de rendre visible une nouvelle catégorie d'usagers aux besoins spécifiques(Pattegay, 2001).

Certains travaux sociologiques adoptèrent cette appellation «jeunes en errance »qui souligne l'aspect subi de leur mode de vie précaire. La fragilité d'une catégorie d'individus de milieu populaire soumise à des logiques complexes de mobilité sociale descendante et inégalitaires explique leur difficulté à se faire une place dans notre société (Déquiré, Jovelin, 2007 ; Langlois, 2014). Au départ, cette terminologie ne concernait que les jeunes accompagnés de chiens qui mendient puis s'étendit à toutes les jeunesses en difficulté présentes dans la rue :tous les jeunes sansabris, les jeunes malades mentaux,les jeunes «qui tiennent les murs » dans les cités (Pattegay, 2001).Cette approche globalisante ne s'intéresse pas aux spécificités de ces jeunesses mais se centre sur la pratique de l'errance interprétée comme le symptôme d'un dysfonctionnement social, familial, psychique(Chobeaux, 2001, Le Rest, 2006 ; Guillou, 1998, Laberge, 2000). Ces travaux optent majoritairement pour une approche diachronique des parcours sociauxet voient dans l'errance juvénile la conséquence d'une éducation familialedéfaillante, d'expériences scolaires chaotiques,de changements sociaux qui engendrent une perte de repère et une diminution forte des chances d'insertion sociale pour une jeunesse issue de milieu populaire en voix de précarisation (Le Rest, 2006 ; Langlois, 2014 ; Chobeaux,2001).

L'approche de l'errance juvénilesous influence objectiviste prêche implicitement pour des méthodes d'enquête distanciée. Les observations s'effectuent davantage auprès des participants qu'avec eux, dans des lieux du travail socialou dans la rue, aux environs des festivals pour une frange de ces jeunes en errance, ceux qui s'appellent eux-mêmes « les zonards », mais rarement là où ils vivent (Chobeaux, 2001; Déquiré, Jovelin, 2007; Guillou, 1998). Réputés violents, délinquants, fragiles psychologiquement, les observations s'axent sur leurs consommations de drogues, leurs comportements et leur rapport aux institutions, intègrent des entretiens ou des questionnaires sur leurs passés familial, scolaire, social. La conduite d'entretien est semi-directive, l'observation courte et distante s'organise en passant d'un groupe de jeunes à un autre dans la journée. N'observant pas les mêmes individus et leurs pratiques sur la durée, les analyses s'arrêtent sur des faits sensationnels dont le système de sens n'est pas révélé (Geertz, 1983). Les consommations de drogues sont interprétées comme une manière d'éviter une souffrance psychique, d'accepter les conditions de vie précaires, comme une quête ordalique ;la violence présente entre les jeunes, comme un signe de carence éducative, de trouble psychologique (Chobeaux, 2001 ;Laberge, 2000).Les recherches s'attellent à explorer leurs passés sans prêter attention à leur quotidien et à leurs systèmes culturels de production de sens. L'espace académique de l'errance juvénile, marqué par la culture du travail social etune sociologie objectivisteest structurée par une distance professionnelle et (ou) épistémologique. Le fait de considérer le sujet errant comme le jouet de forces inconscientesexplique cette tendance à opter pour un regard extérieur et à éviter ainsi toute « pollution interprétative » indigène qu'induirait une posture d'observateur plus participante.

Or, les premiers entretiens réalisés lors d'un travail d'initiation à la recherche auprès de Zonards et les espaces dans lesquels cette enquête sur les parcours se 
déroula me poussèrent à interroger ces conclusions et la distance méthodologique prônée.

\section{LE SQUAT UN ESPACE INTIME IMPLIQUANT}

Cette réflexion épistémologique débuta dès les premiers entretiens réalisés avec des Zonards qui vivaient pour certains en squat, pour d'autres en camion. L'enquête centrée sur leurs pratiques délinquantes etleur mode de vie déviant, j'avais décidé de me concentrer sur les motifs biographiques qui les expliquaient. Je n'avais pas interrogé l'incidence de leurs espaces de vie sur ceux-ci et j'avais donc écarté les lectures propres aux squats (Coutant, 2000).

Il m'était apparu qu'un lien existait entre la «Zone » et le monde des free parties ${ }^{6}$ et que leursparcours étaient dus au rejet d'une méritocratie illusoire. Ces jeunes n'avaient de cesse de revendiquer leur affranchissement des normes légitimes de réussite sociale auquel leur mode de vie, la culture techno à laquelle ils s'affiliaient leur permettaient d'accéder. Mes lectures et mon expérience d'éducatrice en addictologie me poussaient à considérer ces propos comme des rationalisations permettant aux acteurs d'accepter leurs conditions de vie précaires. Cet espace théorique et ma socialisation professionnelle agissaient sur mes premières analyses relativement déterministes et misérabilistes ainsi que sur la manière dont je conduisais mes observations.

En 2008, je fus invitée par Nia, un zonard, à venir dans le squat où il vivait avec sept autres jeunes. Je précisai à Niaque l'observation s'étalerait sur deux périodes de 6 mois à hauteur de trois journées par semaine et Nia négocia mon entrée auprès des habitants du squat où il vivait. J'optai pour une posture d'observateur distante afin de répertorier leurs activités délinquantes, leur organisation grâce à l'usage d'une grille d'observation. La neutralité de mes analyses et ma capacité d'observation étaient à ce moment-là, pour moi, dépendantes d'une observation extérieure. Cette conception de l'observation étaiten partie due à ma socialisation au sein de l'espace académique « jeunes en errance »et à celle liée à mon passé d'éducatrice qui me défendait de trop m'impliquer. Il fallait, pensai-je,pour préserver mon objectivité, que je rompe avec les interprétations «indigènes », que je me dégage des interactions qui auraient pu contaminer mes analyses en instillant des interprétations imprégnées de l'illusio des acteurs (Bourdieu, 1994). Pour moi,seul le chercheur, grâce à la réfléxivité acquise par la mise à distance et ses connaissances théoriques, étaiten mesure d'émettre des analyses objectives et mettre à jour les dominations, les motifs d'action échappant aux acteurs. Si je voulais percevoir objectivement les pratiques déviantes, il fallait que la rupture épistémologique se traduise par une observation distanciée.

Le squatétait constitué de deux maisons mitoyennes et d'un jardin commun. Les fonctions des espaces de la première maison restaient classiques : un espace commun, le salon où tous dînaient ensemble, trois chambres (dont l'une dans le garage), où deux couples et Nia dormaient, une cuisine et une salle de bains. La seconde maison ne comportaitque des chambres. Tous les habitants se servaient des espaces collectifs de la première maison. Cette répartition des espaces allait me contraindre dans mes observations, qui, durant 1 mois,se déroulèrent dans le salon et la cuisine.Tendant à rester extérieure, de par mon choix méthodologique eteffrayée quelque peu par les stéréotypes que j'avais sur la violence dans les squats, je me cachais derrière mon grand carnet de bord etje notais frénétiquement ce que je voyais, j'entendais. Cette

\footnotetext{
${ }^{6}$ Rave party illégale avec des styles musicaux techno plus undergrounds qu'en club.
} 
pratique ethnographique nourrit évidemment au début une méfiance, une distance. Les squatteurs se sentaient considérés comme des rats de laboratoire. J'exerçais sur eux, sans m'en rendre compte, une violence symbolique.

Je n'entrais jamais dans les chambres, ne savais pas où me placer et comptais, il faut le dire, les heures avant mon départ. Je leur parlais peu, j'avais peur de ne pas maintenir la distance nécessaire à mon objectivité. Les zonards me sentant distante se cachaient dans leur chambre pour réaliser leurs deals, pour discuter de sujets sensibles. Mon objectif de ne pas interférer dans leur réalité était totalementillusoire.L'espace fermé du squat et l'intimité que provoqua le partage de la vie domestique me poussèrent, par politesse tout d'abord,à me dévoiler et à participer aux activités du squat. Je dînais avec eux. J'aidais à la préparation des repas. J'allais avec Nia faire les courses. La parole se libéra alors de leur côté, maispas dans le sens que j'imaginais. Ils me testaient : «nous on n'est pas des rats d'labo. Et puis ça sert à quoi ton truc, là ». Certains tentèrent d'établir une forme de domination masculine en me rappelant ma place de femme par des propos provocateurs. J'étais dans un groupe où le culte de la virilité était très important et conditionnait en partie la hiérarchie. Il fallut donc dans cet espace d'observation confiné que je montre moi aussi que «j'en avais » ou que j'accepte d'être traitée comme les fillesauxquelles le droit à la parole sur les sujets politiques et collectifs était dénié. Je ne pouvais pas accepter cette place qui aurait alimenté les interactions de séductions que certains garçons initiaient et qui entravaient en outre les possibilités d'échange sur leur idéologie dont j'avais besoin pour cette recherche. Je décidaisde me positionner tout d'abord sur le pôle masculin. Je rétorquais grossièrement à toutes les provocations, "chambrais " les garçons subalternes du groupe et m'inclinais,comme les autres membres, devant Yogui, le leader.

La proximité corporelle que l'espace restreint forçait, contribua à mieux cerner les habitudes, les normes du groupe, non pas de manière intellectuelle, mais sous la forme d'une encorporation. Je compris que le fonctionnement du groupe, loin des descriptions anomiques que j'avais lues, était structuré de manière familiale. Yogui occupait un rôle de père, et protégeait les siens, maintenait les normes de vie collectives en décidant et en appliquant des sanctions verbales et physiques. Il pourvoyait avec l'aide d'autres zonards engagés depuis quelques années dans la Zone aux besoins alimentaires des plus jeunes. Certains se dédiaient au maintien et à la création de relations extérieures avec des " outsiders " ${ }^{7}$ »à même de les aider. Ces membres dont Nia faisait partie, s'occupaient de la cuisine, des courses, rassuraient affectivement les nouveaux tels des «mères ». La fonction maternelle n'était pas dans ce groupe liée au genre, ni au sexe des individus mais davantage à leurs compétences empathiques, relationnelles et à leur expertise de la rue.

Une socioanalyse réflexive me permit de repérerdes modifications dans ma façon d'agir etde percevoir la réalité. Ces changements constituaient des révélateurs de normes et de valeurs composants la culture zonarde.Je développais ainsi une manière d'être directe et dure. Je m'alignais sur des formes d'interactionsqui permettaient de légitimer ma place dans le groupe. Bien que je tentai de me situer dans un rôle masculin, mon sexe me contraignait à me voir attribuer par les zonards une place de

\footnotetext{
${ }^{7}$ Le terme « Outsiders » désigne les individus ordinaires que les zonards ne nomment pas ou qu'ils appellent «bourgeois» lorsqu'ils les critiquent fortement. Ils sont considérés comme hors du monde zonard. L'appellation " normaux », que j'utilise comme synonyme, permet de souligner l'impact de la logique stigmatique entre les zonards et les acteurs non-zonards.
} 
femme. A l'instar des femmes qui devaient prouver par leurs attitudes non aguicheuses envers les hommes leur qualité de zonarde vertueuse, je rétorquais avec provocation à tout propos sous-entendant une forme de séduction. Je compris que la place, le rôle des femmes s'alignaient sur des valeurs traditionnelles. Je négociais ainsi avec certaines normes féminines et masculines zonardes une place qui me permettait de composer avec ce que j'étais — une femme — et d'obtenir des informations relevant des deux sphères genrées.

L'espace du squat, le temps que j'y passai ${ }^{8}$ nous força à échanger davantage, à nous dévoiler. Pour faciliter une confiance naissante, je les invitai à consulter mon carnet de bord, bien qu'il contienne des réflexions stéréotypées et fasse état de mes craintes. Investis dans sa lecture, six d'entre eux me questionnaient régulièrement, ajoutaient des éléments, corrigeaient certaines erreurs. J'amenai des informations sur des idéologies politiques que je pensais proches des leurs. Nous débattions à partir de mes lectures sur l'anarchie socialiste et primitiviste. Grâce à cela, j'accédaià une place hiérarchiquement valorisée, car j'étais détentrice de savoirs qu'ils n'avaient pas et qui s'accordaient avec leur utopie. Je n'hésitai plus à souligner certains paradoxes entre leurs discours et la réalité. Des débats autour du rejet des hiérarchies sociales et leur fonctionnement pourtant hiérarchisé avec la présence du leadership relativement autocratique de Yogui,s'engagèrent. Ils me permirent de comprendre que ce n'était pas le leadership lui-même qu'ils refusaient mais sa forme désincarnée et illégitime car non basée sur un savoir pragmatique, une gestion du bien-être collectif - qu'ils ne supportaient pas. Contrairement à certaines analyses voyant dans leur culture «un vernis qui cache une réaction vitale de prestance qui est un habillage désespéré $d u$ mal-être profond qui les fait souffrir et qui les a conduits à fuir et à se fuir. ", une pensée politique, une histoire du mouvement, une vision de ce qu'ils souhaitaient comme société existaient(Chobeaux, 2001, p. 15). Elles constituaient des repères culturels qui dictaient leurs conduites ${ }^{9}$.Il fallait, pour les zonards, revenir à un fonctionnement tribal où l'autorité du décideur devait conjuguer charisme, rationalité et tradition. Yogui possédait ces attributs. Il savait défendre les siens, maîtrisait les pratiques zonardes de survie, possédait " un capital guerrier », des connaissances sur la culture techno alternative,le punk et l'anarchisme qui fondaient leurs valeurs, jouissait d'un talent d'orateur et incarnait la figure du « sage expérimenté » de par ses 12 ans de rue et ses nombreuses incarcérations (Sauvadet, 2006).

Les jeunes s'investirent de plus en plus dans l'enquête. Ils me conduisirent dans les appartements de leurs amis, me donnèrent des photographies, se confièrent. Cette ouverture des espaces privés d'observation eut une influence décisive sur mon sujet de thèse. Je décidais non plus dem'intéresser à leurs activités délinquantes en explorant diachroniquement leurs parcours, mais d'aborder leurs biographies, leurs carrières de zonardsen tentant de comprendre comment leurs socialisations plurielles passés et présentes, les interactions avec d'autres groupes sociauxpouvaient conduire à des engagements plus ou moins intenses dans ce mode de vie marginal (Lahire, 2001 ; Grossetti et al, 2010; Becker, 1985).Dans la Family ${ }^{10}$, les manières d'être zonard étaient différentes. Certains vivaient en appartement et ne voulaient surtout pas s'installer en squat, renoncer à une insertion professionnelle, même si celle-ci était uniquement conçue comme un moyen de subvenir à leurs besoins ; d'autres rejetaient tout habitat sédentaire et emploi car ils y voyaient une forme d'aliénation.Ce que

\footnotetext{
${ }^{8}$ Entre $9 \mathrm{~h}$ et $12 \mathrm{H}$ par jour, deux à trois fois par semaine pendant 6 mois.

${ }^{9}$ Voire Pimor (2014).

${ }^{10}$ Nom de la famille de rue observée.
} 
j'avais lu ne correspondait tellement pas à ce que j'observaisqu'il me semblait primordial d'analyser leurs parcours de vie en me débarrassant de mes héritages académiques et de mon ancienne place d'éducatrice; d'aborder cette recherche de manière inductive. J'entrepris alors de réaliser des récits de vie sous la forme d'entretiens. L'implication des zonards mise dans les récits de vie, dans les discussions, planta les graines d'une possible démarche d'ethnographie collaborative, sans que j'en ai alors réellement conscience.

\section{HORS DU SQUAT : ENTRE OBSERVATION MILITANTE ET OBJECTIVATION}

\section{L'observation dans la rue et la stigmatisation zonarde}

Plusieurs éléments expliquent le passage d'une observation extérieure à celle d'une «observation collaborative ». Cette démarche se mit en place du fait de la proximité spatiale que le squat impliquait, de l'engagement du groupe de squatteurs dans l'enquête et de la découverte d'une manière d'aborder la recherche plus inductive et collaborative.L'espace académique contraignant au départ devint, par ouverture à l'anthropologie, à des lectures moins centrées sur les jeunes en errance, un espace d'innovation méthodologique.

A cette époque, j'avais entre les mains l'ouvrage de Clifford et Marcus (1986), «Writting culture » qui me fit sauter le pas de la recherche collaborative. Les auteurs militaient pour que le texte ethnographique devienne un écrit à trois voix : celle du chercheur, celle des enquêtés et celle du lecteur. La postface du Hobo (1993) d'Olivier Schwartz, qui expliquait l'intérêt de combiner le savoir indigène et le savoir scientifique dans l'interprétation des modes de vie marginaux, confirma l'intérêt de me risquer à l'exercice d'une approche collaborative avec les enquêtés. Pour autant, s'il s'agissaitd'une recherche participative, je ne désirai pas réaliser une recherche action et ceux, malgré mon passé d'éducatrice en addictologie qui aurait pu m'y enjoindre. L'objectif de cette recherche collaborative n'était pas de proposer des modalités d'action et d'agir en vue d'améliorer les conditions de vie des zonards ou encore de modifier avec eux leurs pratiques — il m'est toujours apparu épistémologiquement difficile d'endosser conjointement les fonctions incombant aux chercheurs, aux experts et aux travailleurs sociaux - mais d'opter pour un populisme méthodologique qui permette de repérer des rationalités et des logiques zonardes (Olivier De Sardan, déf; Despagné et al, 2001). Puis au cours de ma thèse Florence Bouillon (2009) publia Les mondes du squatetje découvris l'ouvrage d'Isabelle Coutant (2000) Politiques du squatdont les analyses s'appuient sur la subjectivité des squatteurs et sur une observation impliquée du quotidien de squatteurs dans leurs habitats. Ces lectures et la posture engagéedes zonards dans la recherche m'encouragèrent à élire des modalités d'enquête participatives.

L'espace restreint et privé du squat y concourait également car iljouait sur notre relation qui s'intensifia. Si je n'étais pas devenue une des leur, je devins une «amie »écrivaine d'un épisode du squat. Cette complicité me mena à une participation observante en accord avec le principe de collaboration ethnographiqueentre enquêtés et chercheur (Wacquant, 2002). Ma participation ne se situait pas forcément dans le cadre des pratiques proprement zonardes telles que le deal, la consommation de drogue, les bagarres. Je me rendis tout de même complice d'un vol de sac de courses dans la rue. J'acceptais d'exécuter la demande afin de m'intégrer davantage au groupe. En dehors de cet épisode, ma participation se bornait 
à ce que je savais faire et désirais et permit des échanges plus profonds sur nos visions, nos rapports au monde.

Cette participation observante résultait aussi de l'observation conduitedans des espaces publics : la rue, les magasins. Je mendiais, me promenais, faisais les courses avec eux et je prenais par à leur rendez-vous avec d'autres zonards dans la rue. J'assistai à des interactions dévalorisantesde la part des "normaux» envers les zonards, entre autres à des contrôles de police ciblés.

« Nous nous rendons sur une esplanade de la ville où jaillit une fontaine, lieu assez cossu, pour y baigner les chiens. Cinq minutes après nous être assis, deux mobylettes de la police municipale arrivent. Les agents contrôlent les identités des zonards, pas la mienne, leur distribuent à chacun une contravention et nous exhortent à partir. »

Je fus révoltée du traitement qu'ils subissaient, d'autant plus qu'au fur et à mesure, ma tenue s'alignant sans m'en rendre compte sur la leur, je commençaisà être touchée par la stigmatisation et je surinterprétai les regards des « outsiders »dans un sens dénigrant. Je m'alignai sur leurs interprétations et j'avais la sensationd 'être un peu des leurs.

« Il est minuit, je rentre chez moi. Nia m'accompagne à l'arrêt de bus avec ses chiens. Le bus arrive, les chiens montent avec moi. Nia s'excuse auprès du chauffeur et tente de les faire descendre. Le chauffeur souffle, nous regarde peu aimablement. Il m'observe dans son rétroviseur et ne répond pas quand je lui dis au revoir. Il pense que je suis des leurs et l'on voit « la contamination stigmatique » à l'œuvre. »

La stigmatisation ressentie in vivo me poussa à vouloir leur donner un espace de parole plus important et à faire état de leur réflexivité. Je m'aperçus que les parcours biographiques zonards que j'avais repérés n'étaient pas le fruit unique de socialisations primaires mais aussi d'interactions spécifiques dans leur groupe et avec les groupes sociaux extérieurs qu'ils côtoyaient. Un fort sentiment d'appartenance à une communauté était palpable, bien que la dépendance à la Zone soit différente suivant les parcours biographiques.L'étiquetage social qu'il subissait semblait jouer sur la forme communautaire que prenait la Zone et sur l'engagement des acteurs; par conséquent, il fallait que j'étende mon terrain de rechercheaux lieux où cette labellisation se déroulait et aux acteurs non zonards qui y participaient.

\section{Diversifier les espaces, les manières d'observer}

$\mathrm{Du}$ fait des manifestations stigmatiques que les Zonards rencontraient, de leur répercussion sur les parcours biographiques zonards, ma pratique de l'observation s'étendit aux publics des commerçants et des riverains. Sentant que je devenais trop partisane en faveur des zonards, que le populisme méthodologique engendrait un populisme idéologique, il m'apparut utile de diversifier mes lieux d'observations et surtout les publics (Grignon, Passeron, 1989). Rester uniquement présente dans les espaces zonards, surtout celui du squat, dirigeait de toute évidence mes analyses. La socialisation par frottements qui s'y effectuait me poussait à une forme de conversion à la culture zonarde qui, si elle était essentielle pour mon intégration et la compréhension de leurs valeurs et normes, était aussi un biais dans l'analyse (De Singly, 2000).Influencée par le concept de carrière d'Howard. Becker (1985), je réalisais pourtant qu'il n'y avait pas que l'apprentissage des pratiques, des normes, 
des valeurs et de l'histoire zonardes qui provoquaient l'avancée dans leur carrière. L'étiquetage social, souvent lié à des tensions entre les zonards et les « outsiders »basées sur des intérêts et des interprétations de la réalité divergents, à des situations et des contextes particuliers y contribuaient.

Si je voulais déconstruire les stéréotypes sur les zonards, je ne désirais pas pour autant enjoliver la vie zonarde. Leur vie était dure, la violence présente, les paradoxes existants. Ainsi, bien que revendiquant leur rejet de la domination, ils n'hésitaient pas à user de la force pour asseoir leur autorité, par exemple lorsqu'un zonard les avaient rouler lors d'un deal ou transgresser les règles de vivre ensemble. Ils bénéficiaient d'aides sociales, de la charité des "normaux »pour survivre alors qu'ils rejetaient le modèle de notre société et proclamaient leur envie de liberté. Leur réflexivité, la rationalité de leurs pratiques, tout comme celles des autres membres de notre société, n'étaient pas donc aussi importantes que ce que je pensais.

Faire appel aux autres acteurs avec qui ils interagissaienthors du squat pour identifier les causes et la manière dont l'étiquetage déviant se produisait se révélait nécessaire. Certes, les zonards étaient discriminés, mais les "normaux » qu'ils rencontraient sur le lieu de sociabilité, situé en plein centre-ville, pâtissaient de leur présence. Des tensions entre ces deux groupes naissaient dans cet espace et jouaient sur leur engagement dans la Zone. Si la recherche collaborative tend à faire état au plus prés des interprétations des enquêtés principaux, elle doit aussi négocier avec celles du chercheur et des autres acteurs impliqués dans le phénomène étudié. Il s'agissait, dans une réflexivité conjointe, de créer une «zone interprétative » (Desgagné, 2001) autour de pratiques pour développerdes connaissances qui répondaient aux aspirations des diverses parties : celles du chercheur en quête de dévoilement, celles des zonards qui désirent déstigmatiser et faire reconnaître leur mode de vieet celles des « normaux »qui cherchent un vivre ensemble plus apaisé.

Dans la rue, mon observation se concentra sur les interactions entre les passants, les commerçants et les zonards. Elle se situa à deux niveaux: celui duface-à-facedirect entre zonard et «outsiders »dans les situations de mendicité, de regroupement zonard ; celui plus flottant qui me permettait de relever les réactions plus distantes de la part des «normaux » qui n'étaient pas en interaction directe avec les zonards. Autant l'observation des interactions de face-à-face était aisée, autant celle des individus passant plus loin de nous était difficile. Le volume sonore des activitészonardes, les allées et venues, parasitaient ma perception. Un effort de concentration important s'imposait dans la rue, espace vaste de circulation. Il fallait que je puisse détecter sur le visage des passants des signes montrant leur aversion, leur sympathie, leur indifférence envers les zonards, que je remarque leur posture corporelle, leur façon de se déplacer dans l'espace. Changeaient-ils de trottoir à la vue des zonards ? Leur jetaient-ils des regards désapprobateurs ? Pour parvenir à relever ces éléments, je conjuguais deux types d'observation : l'une extérieure où je scrutais les signes non-verbaux de tous les acteurs, l'autre participante qui me permettait de ressentir émotionnellement les réactions des «normaux» en me conformant à l'identité sociale de zonarde qu'ils m'attribuaient — percevoir le langage non-verbal de manière objective n'est pas toujours aisée ; nos sensations peuvent alors compléter ces manques. Lors d'une initiation à la mendicité, Kundevitch, l'un des squatteurs, m'amena devant une boulangerie d'une banlieue populaire de Bordeaux. Contaminée par le stigmate zonard que Kundevitch endossé, je perçus à l'opposé de mon expérience avec Nia en plein centre-ville de Bordeaux que les passants ne semblaient pas effrayés par notre présence, ni agacés. Les interactions explicites peu nombreuses n'indiquaient rien, mais le climat serein me faisait comprendre qu'une cohabitation 
entre zonards et «normaux » était possible. Une fois cette sensation identifiée, je pris physiquement du recul, laissai Kundevitch mendier seul et j'observai les mimiques, la circulation des passants. Ils souriaient, passaient près de lui

Concernant les commerçants qui s'étaient plaint des zonards par voie de presse, l'observation dans la rue ne donnait rien. Ils ne sortaient que rarement de leur magasin et n'interagissaient pas avec les jeunes. Ils appelaient directement la police municipale. Je dus alors assister à des réunions organisées par l'association des commerçants du quartier ou par la mairie, au sujet de l'implantation d'une nouvelle structure sociale destinéeaux zonards. Ne parlant pas, posté en arrière-plan comme d'autres individus, je pus relever des propos assez virulents à l'encontre des jeunes : "Moi, je les enverrais par-dessus bord dans la rivière! " et comprendre la posture, les représentations des commerçants et des riverains.Pour obtenir plus de données, je décidai de réaliser des observations dans les commercesde manière assez distante afin de ne pas gêner la clientèle ; ce qui aurait entaché l'acceptabilité de la recherche déjà mise à mal par le contexte. Je notai ainsi que devant la clientèle, les commerçants les moins à l'aise avec les zonards évitaient d'interagir avec eux. Ils ne voulaient pas s'emportaient et nuire à leur image. Je décidai d'effectuer des entretiens avec des commerçants pour mieux saisir leurs visions des zonards et ce qui expliquaient les différences de comportements à leur égard. Fatigués par la situation problématique des regroupements de zonards, habités par le sentiment de ne pas être entendus par les pouvoirs publics, la méfiance, la lassitude générèrent beaucoup de refus d'entretiens et empêchèrent d'établir un travail collaboratifavec les commerçants. Cinqcommerçants, dont quatre qui entretenaient des relations cordiales avec les zonards et le président de l'association commerçante moins clément, m'accordèrent des entretiens.

Le croisement d'outils fut pertinent, car les entretiens me donnèrent accès à leurs perceptions des jeunesses précaires, de notre société, des politiques, de l'engagement des pouvoirs publics sur le vivre ensemble. Les discours des quatre commerçants étaient ambivalents, car s'ils appréciaient certains jeunes, les exactions d'autres zonards empêchaient qu'ils les défendent. Les préjudices commerciaux qu'entraînait la présence de ces jeunes qui faisait fuir la clientèle ne furent que peu abordés si ce n'est par le Président de l'association qui lança une pétition afin que les pouvoirs publics interviennent.Il me raconta comment après avoir averti à de multiples reprises la mairie et la police, il se chargeait lui-même de les faire fuir en sortant avec une barre à mine, comment les vigiles du supermarché les délogeaient à coup de seaux d'eau. Les autres, beaucoup moins offensifs, voyaient les zonards comme des jeunes dont la famille était défaillante et qui ne pouvaient trouver d'emploi dans un contexte économique de crise.Toutefois, ils reprochaient à certains leur sans-gêne, leur manque de réflexion et leurs activités illégales. Ils ne défendaient jamais pour autantles commerçants qui s'étaient regroupés pour les chasser ou qui appelaient la police. La solution était contenue dans un dialogue à établir entre eux et les zonards rendant nécessaire la mise en place d'une médiation à laquelle les pouvoirs publics devait participer. En effet, les commerçants et les zonards qui s'entretenaient régulièrement avaient trouver ensemble un terrain d'entente. La buraliste les autorisait ainsi à mendier devant sa boutique en échange de quoi ils devaient se montrer aimable avec la clientèle. Pour la remercier, les zonards sortaient son chien. Je constatai que le fait de se connaître, sans pour autant se ressembler, générait des interactions d'entraide des deux côtés, un respect des différences de points de vue que je ne pensais pas observer. Les jeunes réalisaient ainsi les courses d'une commerçante âgée et elle, leur 
avançait des paquets de cigarettes. Ils protégeaient aussi ces commerçants des zonards peu scrupuleux.

\section{CONCLUSION : LES ESPACES D'OBSERVATIONS DES CONTRAINTES INNOVANTES}

L'espace scientifique et professionnel de l'errance juvénile m'a conduit à défendre dans un premier temps l'utilité d'une observation "froide » que mon expérience de l'espace zonard est venue percuter. L'espace privé du squat m'a enjoint à opter pour une posture participante. Il a instillé dans mon esprit la nécessité d'associer les participants à cette enquête en leur reconnaissant une place de « sociologue indigène ». La rue, les espaces mixtes socialement ont affirmé l'utilité de cette collaboration dans l'analyse de l'influence de l'étiquetage social sur les carrières zonardes. Cependant, ces lieux ont aussi provoqué chez moi une remise en cause de ma posture trop militante de la cause zonarde et m'ont guidée vers une ouverture à d'autres populations.Ces espaces hors-zone ont soulevé de nouvelles interrogations et ont requis de nouvelles adaptations méthodologiques. Cette posture engageant à multiplier les manières d'observer n'est évidemment pas sans lien avec la démarche inductive de cette recherche. L'induction, sensible aux espaces, aux populations,conduit, me semble-t-il, à une malléabilité plus grande des outils et des postures.

C'est ainsi en souscrivant à une adaptabilité méthodologique de l'observation que de nouveaux résultats sur le mode de vie et les parcours biographiques zonards ont pu naître. Le «jonglage » avec des types d'observation et des espaces différents, m'a permis de saisir que le seul passé des jeunes, aussi douloureux soit-il, n'est pas la seule cause de leur engagement zonard. J'ai pu constaté, grâce à une « observation collaborative » conduite dans des espaces privés zonards, qu'il n'y a avait pas que les socialisations familiales, de classe populaire, scolaire qui conditionnaient la vie dans la Zone mais aussi des postures éthiques nées d'expériences qui avaient provoqué chez les acteurs une réelle réflexion sur la place qu'ils désiraient prendre dans notre société. Des rencontres avec des groupes de pairs baignant dans une culture alternative comme celle des redskins, des punks, des expériences professionnelles, universitaires décevantes ont permis aux acteurs de percevoir la domination sociale, l'illusion méritocratique dans lesquelles il était pris et de réfléchir à une autre forme de faire société. Certains parcours biographiques conduisent à une implication totale dans la vie déviante zonarde, au franchissement des étapes qui jalonnent la carrière zonarde, d'autres, au contraire, restreignent les opportunités de passage à des séquences de carrière zonarde plus impliquées (Becker, 1985). Cette bifurcation vers la zone tient par ailleurs à la rencontre avec des teuffeurs ${ }^{11}$ et à la fréquentation de Free parties qui offrent un nouvel univers à des jeunes en quête « d'autre chose » (Grossetti et al, 2010). L'engagement dans la Zone requiert des apprentissages, une implication subjective et affective évidente. La forme familiale du groupe de la Family, les liens affectifs encouragent l'engagement des jeunes. Les observations dans la rue avec les commerçants, les passants $m$ 'ont permis de comprendre que l'engagement zonard est entretenu par des interactions avec des out- groups qui poussent ces jeunes à réaffirmer, par un retournement stigmatique, leur identité zonarde en polarisant des pratiques jugées déviantes dans l'espace public. Ces interactions s'additionnent à une histoire zonarde fictionnelle commune qui se nourrit de leurs expériences individuelles et de l'histoire «des techno travellers » auxquels ils s'identifient pour

\footnotetext{
${ }^{11}$ Adeptes des free parties.
} 
établir des frontières entre les deux groupes - les zonards et les "normaux ». Ces frontières poussent ainsi les zonards à un engagement dans la Zone, sa culture et ses pratiques. Ainsiles parcours biographiques zonardes se caractérisent par un mélange de logiques éthiques, socialisatrices et interactionnelles qui invite à penser leur étude de manière complexe. La description de la Zone que j'ai réalisée grâce à «l'observation collaborative» menée dans le squat autorise à entendre que si on s'y meurtrit souvent, la Zone ne peut être uniquementconsidérée comme le symbole d'une démission sociale de la part de cette jeunesse. Dans la Zone, on rit, on crée de vraies amitiés, on innove, on débat, on s'implique dans la politique. Y voir uniquement le terminus du désespoir ou la finalisation d'un processus d'exclusion, c'est succomber au piège de l'ethnocentrisme.

BECKER, H.S. 1985. Outsiders, Paris, Métailié.

BLONDET in BENSA, A \& FASSIN, D. (Dir.). 2008. Les politiques de l'enquête, épreuves ethnographiques, Paris, La découverte.

BOUILLON, F. 2009. Le monde des squats, Paris, PUF.

BOURDIEU, P. 1994. «Un acte désinteressé est-il possible?», Raisons pratiques, Paris, Seuil.

CHOBEAUX, F. 2001. L'errance active, Politiques publiques et pratiques professionnelles, Paris, La Découverte.

CLIFFORD, J \& MARCUS, G. E. 1986. Writing culture : the poetics and politics of ethnography : a school of American research advanced seminar, Los Angeles, London, University of California Press.

COUTANT, I. 2000. Politiques du squat, Paris, La dispute.

DEQUIRE, A\& JOVELIN. E. 2007. « Des jeunes dans la tourmente. Les jeunes sans domicile fixe à l'épreuve de la rue », Pensée plurielle , n¹4, p. 125-147.

DESGAGNÈ, S ; BEDNARZ, N ; LEBUIS, P ; POIRIER, L \& COUTURE, C . 2001. «L'approche collaborative de recherche en éducation : un rapport nouveau à établir entre recherche et formation ", Revue des sciences de l'éducation, vol 27, ${ }^{\circ} 1, \mathrm{p} .33$ 64.

FAVRET-SAADA, J. 1985, Les mots, la mort, les sorts, Paris, Gallimard.

GEERTZ, C. 1983. Bali : interprétation d'une culture, Paris, Gallimard.

C. GRIGNON \& J.-C. PASSERON. 1989. Le Savant et le populaire. Misérabilisme et populisme en sociologie et en littérature, Paris, Le Seuil.

GROSSETTI, M ; BESSIN, M \& BIDART, C (Dir.). 2010.Bifurcations. Les sciences sociales face aux ruptures et à l'évènement, Paris, La Découverte.

GUILlOU, J. 1998. Les jeunes sans domicile fixe et la rue ou «Au bout d'être énervé », Paris, L'harmattan.

KUHN, T. 1962. La Structure des révolutions scientifiques, Flammarion

LABERGE, D. 2000.L'errance urbain, Québec, éd Multimondes.

LE REST, P. 2006. L'errance des jeunes adultes, causes, effets, perspectives, Paris, L'harmattan.

LAHIRE, B. 2001. L'homme pluriel: les ressorts de l'action, Paris, Hachette Littteratures.

LANGLOIS, E. 2014. « De l'inconvénient de n'être le problème de personne : cécité institutionnelle et vulnérabilité sociale des jeunes en errance », Pensée Plurielle, $\mathrm{n}^{\circ}$ 35, p. 83-99.

OLIVIER DE SARDAN, J. P. 2008. La rigueur du qualitatif : Les contraintes empiriques de l'interprétation socio-anthropologique, Paris, Accadémia. 
OLIVIER DE SARDAN, J. P. Déf. De l'amalgame entre analyse-système, recherche participative et recherche-action, et de quelques problèmes autour de chacun de ces termes, conférence en ligne, http://horizon.documentation.ird.fr/exldoc/pleins_textes/divers 11-03/010009948.pdf

PATTEGAYY, P. 2001. «L'actuelle construction, en France, du problème des jeunes en errance ", Déviance et société , $\mathrm{n}^{\circ} 25$, p. 257-277.

PIMOR,T. 2014. Zonards, Paris, PUF.

SAUVADET, T. 2006. Le capital guerrier, Armand Collin.

SCHWARTZ, O, in Anderson, N.1993. Le hobo, Sociologie du sans-abri, Paris, Nathan.

SINGLY (DE), F. 2000. Libres ensemble, L'individualisme dans la vie commune, Paris, Nathan.

WACQUANT, L. 2002. Corps et âme, Carnet d'un apprentis boxeur, Paris, Agone. 